\title{
132. Some Notes on Cesàro Summation
}

\author{
By Kenji Yano \\ Department of Mathematics, Nara Women's University, Nara, Japan \\ (Comm. by Z. SuetunA, M.J.A., Dec. 12, 1959)
}

In this paper we shall establish two lemmas concerning the Cesàro summability of Fourier series. Of these, Theorem 1 is closely related to the result of Chandrasekharan and Szász [2, Theorem 5]. And Theorem 2 is concerned with the estimation of the principal part of Fejér kernels.

1. THEOREM 1. If $\varphi(t) \in L$ in $0 \leqq t \leqq t_{0}$, and $r>0, \delta>0$, and $q$ be arbitrary, then

$$
\Phi_{r}(t) \equiv \frac{1}{\Gamma(r)} \int_{0}^{t}(t-u)^{r-1} \varphi(u) d u=o\left(t^{q}\right) \quad(t \rightarrow 0)
$$

is equivalent to

Letting

$$
\Phi_{r}^{\delta}(t) \equiv \frac{1}{\Gamma(r)} \int_{0}^{t}(t-u)^{r-1} u^{\delta} \varphi(u) d u=o\left(t^{q+\delta}\right) \quad(t \rightarrow 0) .
$$

$$
\varphi_{r}^{\delta}(t)=\frac{\Gamma(r+\delta+1)}{\Gamma(\delta+1)} t^{-(r+\delta)} \Phi_{r}^{\delta}(t) \quad(\delta \geqq 0),
$$

and $\varphi_{r}(t)=\varphi_{r}^{0}(t)$, we have the following

Corollary 1. Let $\varphi(t) \in L$ in $\left(0, t_{0}\right)$, and $r>0, \delta>0$, and $q$ be arbitrary. Then

is equivalent to

$$
\varphi_{r}(t)=s+o\left(t^{q-r}\right) \quad(t \rightarrow 0)
$$

$$
\varphi_{r}^{\delta}(t)=s+o\left(t^{q-r}\right) \quad(t \rightarrow 0),
$$

where $s$ is a constant independent of $t$.

Concerning this corollary, cf. loc. cit. [2].

We need two lemmas:

Lemma 1. Theorem 1 holds when $\delta=k$, where $k$ is a positive integer.

This is Lemma 3 in the paper [3], but for the sake of completeness we prove it. We first consider the case $k=1$. Observe now that

$$
\Phi_{r}^{1}(t)=t \Phi_{r}(t)-r \Phi_{r+1}(t),
$$

and that necessarily, since $r>0$,

$$
\Phi_{r+1}(t)=o\left(t^{r}\right) .
$$

If $q>-1$, then (1.1) implies

and then by (1.3),

$$
\Phi_{r+1}(t)=o\left(t^{q+1}\right),
$$

$$
\Phi_{r}^{1}(t)=o\left(t^{q+1}\right),
$$

which follows from (1.1) still when $q \leqq-1$, by (1.3) and (1.4). 
Inversely, (1.6) is written as, by (1.3),

$$
\frac{\Phi_{r}(t)}{t^{r}}-r \frac{\Phi_{r+1}(t)}{t^{r+1}}=o\left(t^{q-r}\right)
$$

i.e.

$$
\frac{d}{d t}\left[t^{-r} \Phi_{r+1}(t)\right]=o\left(t^{q-r}\right)
$$

If $q-r>-1$, integrating both sides from zero to $t$, we have $t^{-r} \Phi_{r+1}(t)$ $=o\left(t^{q-r+1}\right)$, by (1.4), which is equivalent to (1.5). And, (1.5) holds still when $q-r \leqq-1$ again by (1.4). Consequently, (1.6) implies (1.1) by (1.3).

We have thus the lemma when $k=1$. In the general case $k>1$, replacing $\varphi(u)$ by $u \varphi(u), u^{2} \varphi(u), \cdots$, successively it is proved by induction.

LEMMA 2. If $\varphi(t) \in L$ in $(0, x)$ and $0<y<x, 0<r \leqq 1$, then

$$
\left|\frac{1}{\Gamma(r)} \int_{0}^{y}(x-t)^{r-1} \varphi(t) d t\right| \leqq \max _{0 \leqq u \leqq x}\left|\Phi_{r}(u)\right| .
$$

This is due to Riesz [1].

Proof of Theorem 1. (I) The case $0<r \leqq 1, r \leqq q$. By the second mean-value theorem

$$
\begin{array}{rlr}
\Phi_{r}^{\delta+\eta}(t) & =\frac{1}{\Gamma(r)} \int_{0}^{t}(t-u)^{r-1} u^{\delta+\eta} \varphi(u) d u & \\
& =\frac{t^{\delta}}{\Gamma(r)} \int_{\xi}^{t}(t-u)^{r-1} u^{\eta} \varphi(u) d u & (0<\xi<t) \\
& =t^{\delta} \Phi_{r}^{\eta}(t)-\frac{t^{\delta}}{\Gamma(r)} \int_{0}^{\xi}(t-u)^{r-1} u^{\eta} \varphi(u) d u, &
\end{array}
$$

where $\delta>0$ and $\eta \geqq 0$. So, by Lemma 2, we have

$$
\left|\Phi_{r}^{\delta+\eta}(t)\right| \leqq 2 t^{\delta} \cdot \max _{0 \leq u \leq t}\left|\Phi_{r}^{\eta}(u)\right| .
$$

Hence, if $\Phi_{r}(t)=o\left(t^{q}\right)$, then (1.7) with $\eta=0$ yields $\Phi_{r}^{\delta}(t)=o\left(t^{\delta+q}\right)$, since $q>0$. Inversely, if $\Phi_{r}^{\eta}(t)=o\left(t^{q+\eta}\right)$, then (1.7) with $\delta=[\eta]+1-\eta$ yields

$$
\Phi_{r}^{[\eta]+1}(t)=t^{[\eta]+1-\eta} \cdot o\left(t^{\eta+q}\right)=o\left(t^{[\eta]+1+q}\right),
$$

which implies $\Phi_{r}(t)=o\left(t^{q}\right)$ by Lemma 1 , since $[\eta]+1$ is integral. Hence, we get the present case.

(II) The case $1<r \leqq q$. We have the identities

$$
\begin{aligned}
\Phi_{r}^{\delta}(t)= & \frac{(r-1) t}{\Gamma(r)} \int_{0}^{t}(t-u)^{r-2} u^{\delta-1} \Phi_{1}(u) d u \\
& -\frac{r-1+\delta}{\Gamma(r)} \int_{0}^{t}(t-u)^{r-1} u^{\delta-1} \Phi_{1}(u) d u, \\
\Phi_{r}^{\delta}(t)= & \frac{t^{r+\delta}}{\Gamma(r)} \frac{d}{d t}\left(\frac{1}{t^{r-1+\delta}} \int_{0}^{t}(t-u)^{r-1} u^{\delta-1} \Phi_{1}(u) d u\right) .
\end{aligned}
$$

And, (1.1) is equivalent to, since $r>1$, 


$$
\int_{0}^{t}(t-u)^{r-2} \Phi_{1}(u) d u=o\left(t^{q}\right)
$$

Suppose now that the theorem is true when $r$ is replaced by $r-1$. Then, $(1.1)^{\prime}$ is equivalent to

$$
\int_{0}^{t}(t-u)^{r-2} u^{\delta-1} \Phi_{1}(u) d u=o\left(t^{q+\delta-1}\right), \quad \delta>1,
$$

which clearly implies

$$
\int_{0}^{t}(t-u)^{r-1} u^{\grave{\delta}-1} \Phi_{1}(u) d u=o\left(t^{q+\delta}\right) .
$$

Substituting this and (1.10) into (1.8), we have

$$
\Phi_{r}^{\delta}(t)=o\left(t^{q+\delta}\right), \quad \delta>1,
$$

and then $\Phi_{r}^{\delta-1}(t)=o\left(t^{q+\delta-1}\right)$ by Lemma 1 .

Inversely, if (1.2) holds, i.e. $\Phi_{r}^{\delta}(t)=o\left(t^{q+\delta}\right)$ then by integrating, we have (1.11) for $\delta>0$ from (1.9). (1.11) and (1.2) imply (1.10) by (1.8), and then $(1.1)^{\prime}$ by the above assumption. We thus get the present case by induction.

(III) General case $r>0, q$ arbitrary. We put

$$
\psi(u)=u^{k} \varphi(u)
$$

where $k$ is a positive integer such that $k+q>r$, and define

$$
\Psi_{r}^{\delta}(t)=\frac{1}{\Gamma(r)} \int_{0}^{t}(t-u)^{r-1} u^{\delta} \psi(u) d u \quad(\delta \geqq 0),
$$

and $\Psi_{r}(t)=\Psi_{r}^{0}(t)$. Then

$$
\Psi_{r}(t)=\Phi_{r}^{k}(t), \quad \Psi_{r}^{\delta}(t)=\Phi_{r}^{k+\delta}(t)
$$

By the preceding result, we see that

$$
\Psi_{r}(t)=o\left(t^{k+q}\right) \Leftrightarrow \Psi_{r}^{\delta}(t)=o\left(t^{k+q+\delta}\right),
$$

since $k+q>r$. This is the same thing as, by (1.12),

$$
\Phi_{r}^{k}(t)=o\left(t^{k+q}\right) \Leftrightarrow \Phi_{r}^{k+\delta}(t)=o\left(t^{k+q+\delta}\right),
$$

whence follows, by Lemma 1 ,

$$
\Phi_{r}(t)=o\left(t^{q}\right) \Leftrightarrow \Phi_{r}^{\delta}(t)=o\left(t^{q+\delta}\right) .
$$

This proves the theorem completely.

2. THEOREM 2.1. If $0<\delta<1,-1<\beta, 1 \leqq k$ and $0<u<\pi$, then we have

$$
\begin{aligned}
F(u, k) & \equiv \int_{u}^{\pi}(t-u)^{\delta-1}\left(2 \sin \frac{1}{2} t\right)^{-\beta} e^{i k t} d t, \quad i=\sqrt{-1} \\
= & \frac{\Gamma(\delta)}{k^{\delta}}\left(2 \sin \frac{1}{2} u\right)^{-\beta} e^{i(k u+\delta \pi / 2)}+2^{-\beta}(\pi-u)^{\delta-1} \frac{e^{i k \pi}}{i k} \\
& +O\left(\frac{(\pi-u)^{\delta-2}}{k^{2} u^{\beta}}\right)+O\left(\frac{1}{k^{\delta+1} u^{\beta+1}}\right),
\end{aligned}
$$

where $O$ 's are independent of $u$ and $k$.

PROOF. 


$$
\begin{aligned}
F(u, k)= & \left(2 \sin \frac{1}{2} u\right)^{-\beta} \int_{u}^{\pi}(t-u)^{\delta-1} e^{i k t} d t \\
& +\int_{u}^{\pi}(t-u)^{\delta} m(t, u) e^{i k t} d t=\left(2 \sin \frac{1}{2} u\right)^{-\beta} I+J,
\end{aligned}
$$

where $0<u<\pi$, and

$$
m(t, u)=\frac{1}{t-u}\left[\left(2 \sin \frac{1}{2} t\right)^{-\beta}-\left(2 \sin \frac{1}{2} u\right)^{-\beta}\right] .
$$

Here, for the sake of convenience we denote

$$
m(u, u)=\lim _{t \rightarrow u} m(t, u)=\frac{d}{d u}\left(2 \sin \frac{1}{2} u\right)^{-\beta} .
$$

By the mean-value theorem,

And, clearly

$$
m(t, u)=\left.m(u, u)\right|_{u=u_{1}} \quad\left(u<u_{1}<t\right) .
$$

$$
\frac{\partial}{\partial t} m(t, u)=\frac{1}{t-u}[m(t, t)-m(t, u)] .
$$

From these relations we see that $m(t, u)$ conserves a constant sign for $0<u<t \leqq \pi$, and increases with $1 / t$ in absolute value, and that

$$
|m(t, u)|<|m(u, u)|<\frac{K}{u^{\beta+1}},
$$

where and in the sequel $K$ denotes an absolute constant, and it may vary from one occurrence to another. Now,

$$
I=\int_{u}^{\pi}(t-u)^{\delta-1} e^{i k t} d t=\int_{u}^{\infty}-\int_{\pi}^{\infty} .
$$

And

$$
\begin{aligned}
\int_{u}^{\infty} & =\int_{u}^{\infty}(t-u)^{\delta-1} e^{i k t} d t \\
& =e^{i k u} \int_{0}^{\infty} x^{\delta-1} e^{i k x} d x \\
& =e^{i k u} \cdot \frac{\Gamma(\delta)}{k^{\delta}} e^{i \delta \pi / 2},
\end{aligned}
$$

by a well-known classical formula, cf. Zygmund [4, p. 224$]$.

$$
\begin{aligned}
\int_{\pi}^{\infty} & =\int_{\pi}^{\infty}(t-u)^{\delta-1} e^{i k t} d t \\
& =\left[(t-u)^{\delta-1} \frac{e^{i k t}}{i k_{t}}\right]_{t=\pi}^{\infty}-(\delta-1) \int_{\pi}^{\infty}(t-u)^{\delta-2} \frac{e^{i k t}}{i k} d t \\
& =-(\pi-u)^{\delta-1} \frac{e^{i k \pi}}{i k}-(\delta-1) I_{1},
\end{aligned}
$$

and clearly $I_{1}=O\left((\pi-u)^{\delta-2} / k^{2}\right)$. Hence, from (2.6) we get

$$
I=\frac{\Gamma(\delta)}{k^{\delta}} e^{i(k u+\delta \pi / 2)}+(\pi-u)^{\delta-1} \frac{e^{i k \pi}}{i k}+O\left(\frac{(\pi-u)^{\delta-2}}{k^{2}}\right) \text {. }
$$

Next, integrating by parts and using (2.4), 


$$
\begin{gathered}
J=\int_{u}^{\pi}(t-u)^{\delta} m(t, u) e^{i k t} d t \\
=\left[(t-u)^{\delta} m(t, u) \frac{e^{i k t}}{i k}\right]_{t=u}^{\pi}-(\delta-1) \int_{u}^{\pi}(t-u)^{\delta-1} m(t, u) \frac{e^{i k t}}{i k} d t \\
\quad-\int_{u}^{\pi}(t-u)^{\delta-1} m(t, t) \frac{e^{i k t}}{i k} d t \\
=(\pi-u)^{\delta} m(\pi, u) \frac{e^{i k \pi}}{i k}-(\delta-1) J_{1}-J_{2} .
\end{gathered}
$$

By the monotonity of $m(t, u)$, and (2.5),

$$
\left|J_{1}\right|<K \frac{|m(u, u)|}{k^{\delta+1}}<\frac{K_{1}}{k^{\delta+1} u^{\beta+1}} .
$$

It is analogous to $J_{2}$. Hence, from (2.8) and (2.2),

$$
J=(\pi-u)^{\delta-1}\left[2^{-\beta}-\left(2 \sin \frac{1}{2} u\right)^{-\beta}\right] \frac{e^{i k \pi}}{i k}+O\left(\frac{1}{k^{\delta+1} u^{\beta+1}}\right) .
$$

Substituting (2.7) and (2.9) into (2.1) we get the theorem.

Theorem 2.1 may be improved more precisely as follows:

THEOREM 2. If $0<\delta<1,-1<\beta, 1 \leqq k$ and $0<u<\pi$, then

$$
\begin{aligned}
& \int_{u}^{\pi}(t-u)^{\delta-1}\left(2 \sin \frac{1}{2} t\right)^{-\beta} e^{i k t} d t \\
&= \frac{\Gamma(\delta)}{k^{\delta}}\left(2 \sin \frac{1}{2} u\right)^{-\beta} e^{i(k u+\delta \pi / 2)} \\
&+\frac{\Gamma(\delta+1)}{k^{\delta+1}} \frac{d}{d t}\left(2 \sin \frac{1}{2} u\right)^{-\beta} e^{i(k u+(\delta+1) \pi / 2)} \\
&+2^{-\beta}(\pi-u)^{\delta-1} \frac{e^{i k \pi}}{i k}+(\delta-1) \cdot 2^{-\beta}(\pi-u)^{\delta-2} \frac{e^{i k \pi}}{k^{2}} \\
&+O\left(\frac{(\pi-u)^{\delta-3}}{k^{3} u^{\beta}}\right)+O\left(\frac{(\pi-u)^{\delta-2}}{k^{3} u^{\beta+1}}\right)+O\left(\frac{1}{k^{\delta+2} u^{\beta+2}}\right),
\end{aligned}
$$

where $O$ 's are independent of $u$ and $k$.

Proof. We use the notations in the preceding proof. Integrating by parts,

$$
\begin{aligned}
I_{1} & =\int_{\pi}^{\infty}(t-u)^{\delta-2} \frac{e^{i k t}}{i k} d t \\
& =(\pi-u)^{\delta-2} \frac{e^{i k \pi}}{k^{2}}+O\left(\frac{(\pi-u)^{\delta-3}}{k^{3}}\right) .
\end{aligned}
$$

Applying Theorem 2.1 replaced $\left(2 \sin \left(2^{-1} t\right)\right)^{-\beta}$ by $m(t, u)$ to $J_{1}$, and observing that by (2.4) and (2.3)

$$
\left|\frac{\partial}{\partial t} m(t, u)\right|<\frac{K}{u^{\beta+2}}
$$

for $0<u<t \leqq \pi$, we have, as it is easily verified, 


$$
\begin{aligned}
J_{1} & =\int_{u}^{\pi}(t-u)^{\delta-1} m(t, u) \frac{e^{i k t}}{i k} d t \\
& =\frac{\Gamma(\delta)}{k^{\delta}} \cdot \frac{m(u, u)}{i k} e^{i(k u+\delta \pi / 2)}+m(\pi, u)(\pi-u)^{\delta-1} \frac{e^{i k \pi}}{(i k)^{2}}+O(R),
\end{aligned}
$$

where

$$
R=\frac{(\pi-u)^{\delta-2}}{k^{3} u^{\beta+1}}+\frac{1}{k^{\delta+2} u^{\beta+2}}
$$

Similarly

$$
\begin{aligned}
J_{2} & =\int_{u}^{\pi}(t-u)^{\delta-1} m(t, t) \frac{e^{i k t}}{i k} d t \\
& =\frac{\Gamma(\delta)}{k^{\delta}} \cdot \frac{m(u, u)}{i k} e^{i(k u+\delta \pi / 2)}+m(\pi, \pi)(\pi-u)^{\delta-1} \frac{e^{i k \pi}}{(i k)^{2}}+O(R),
\end{aligned}
$$

where $m(\pi, \pi)=0$.

Substituting these relations into the expressions of $I$ and $J$ respectively, (2.1) yields the desired result.

\section{References}

[1] M. Riesz: Sur un théorème de la moyenne et ses applications, Acta Szeged, 1, 114-126 (1922).

[2] K. Chandrasekharan and O. Szász: On Bessel summation, Amer. J. Math., 20, 709-729 (1948).

[3] K. Yano: Convexity theorems for Fourier series I, J. Math. Soc. Japan (to appear).

[4] A. Zygmund: Trigonometrical Series, Warszawa-Lwow (1935). 